CYBERPSYCHOLOGY, BEHAVIOR, AND SOCIAL NETWORKING Volume 14, Number 11, 2011a Mary Ann Liebert, Inc.DOI: 10.1089/cyber.2010.0445

\title{
Are Cyberbullies Less Empathic? Adolescents' Cyberbullying Behavior and Empathic Responsiveness
}

Georges Steffgen, Ph.D.,1 Andreas Ko n̈ig, M.S.,1 Jan Pfetsch, Ph.D.,2 and Andre' Melzer, Ph.D.1

Abstract

Meta-analyses confirm a negative relationship between aggressive behavior and empathy, that is, the ability to understand and share the feelings of others. Based on theoretical considerations, it was, therefore, hypothesized that a lack of empathic responsiveness may be characteristic for cyberbullies in particular. In the present study, 2.070 students of Luxembourg secondary schools completed an online survey that included a cyberbullying questionnaire 4 and a novel empathy short scale. According to the main hypothesis, analyses of variances indicated that cyberbullies demonstrated less empathic responsiveness than non-cyberbullies. In addition, cy- berbullies were also more afraid of becoming victims of cyberbullying. The findings confirm and substantially extend the research on the relationship between empathy and aggressive behavior. From an educational point of view, the present findings suggest that training of empathy skills might be an important tool to decrease cyberbullying. 